\title{
Effects of unilateral upper incisor extraction on facial growth of young rats
}

\author{
Marcos K. Yamamoto ${ }^{1}$, Moacyr D. Novelli² and João Gualberto C. Luz ${ }^{1}$ \\ Departments of Oral and Maxillofacial Surgery ${ }^{1}$ and Pathology ${ }^{2}$, School of Dentistry, University of São Paulo
}

(Recieved 16 June and accepted 10 November 1997)

\begin{abstract}
The effects of unilateral extraction of the upper incisor (one tooth) on facial growth of young rats were studied. The remaining upper incisor showed deviation towards the opposite side, and there was regular dental wear of all the incisors. The effects were evident only in the mid-facial area. Cephalometric measurements showed significant shortening with deviation of the incisor bone on the extraction side. There was no significant disturbance of the growth of the rest of the maxilla and mandible. These results indicate that upper incisor extractions have a localized influence, leading to impairment of incisor bone growth.
\end{abstract}

Key words: facial growth; upper incisor extraction; incisor bone asymmetry.

\section{Introduction}

Postnatal craniofacial growth can be impaired by trauma, dietary composition, and tooth loss. Condylectomy produces a reduction in the length of the mandible in young hamsters (1). Resection of the zygomatic arch in young rats causes increased facial projection and decreased transverse facial width on the same side (2). Also, soft tissue operations, such as incision or partial excision of the upper lip in growing rats can result in anterior deviation of the premaxilla (3).

Administration of a soft diet to young rats influences areas of bone remodelling, resulting in anteriorly directed growth of the viscerocranium and a diminished angular process of the mandible $(4,5)$. Also, a soft diet causes changes in the shape of the mandible due to slowed periosteal bone growth (6). A different dietary consistency and malocclusion induced by extraction of molar teeth influence the development of the masticatory organs, mandibular bone and masseter muscle (7).

Studies have demonstrated the effect of some kinds of extraction on facial development. Extraction of the lower incisor and excision of the temporal and masseter muscles of growing rats result in shortening of the mandible (8). Extraction of the mandibular incisor and molar teeth of young hamsters causes a decrease in size of the

Correspondence to Dr. João Gualberto C. Luz, Rua Duarte de Azevedo 284, s.22, 02036-021 São Paulo SP, Brazil mandibular body, loss of normal occlusion, and a shift of the mandible medially and cranially (1). Removal of all four upper incisors in growing rabbits inhibits the anterior growth of the premaxilla, and removal of both lower incisors causes shortening of the symphyseal area and anterior rounding of the mandible (9). No studies, however, have examined the effect of unilateral extraction of an upper incisor on facial growth in experimental animals.

The purpose of the present study was to analyze the effect of unilateral extraction of a single upper incisor on facial growth in young rats.

\section{Materials and Methods}

The study animals were 35 one-month-old Rattus norvegicus albinus individuals. The upper right incisor was removed under general anesthesia with ether. One tooth was extracted per animal. Syndesmotome and curved mosquito (Halstead) forceps were used for extraction, and a single suture with 5-0 catgut concluded the procedure. All the animals were fed an ordinary diet of rodent feed hard pellets, powdered during the first week postoperatively, and water ad libitum. The rats were sacrificed at three months of age, decapitated, their skins removed, and finally they were fixed in $10 \%$ buffered formalin. Following formalin fixation for one week, axial (dorsal-ventral) and rostro-caudal projections of the skull were obtained, and care was taken so as to mantain the horizontal and vertical planes. The skull was placed directly on a radiographic film, with the inferior border of the mandible as the horizontal plane reference during axial projection, and a cylindrical plastic holder was used to keep the vertical plane reference during rostro-caudal projection. Radiograms were taken with a standard dental machine at $60 \mathrm{kV}$ and $10 \mathrm{~mA}$, with an exposure time of $0.4 \mathrm{~s}$. A constant focus to film distance of $40 \mathrm{~cm}$ was mantained, periapical films being used (Ektaspeed, Eastman Kodak Co.). Subsequently, 10 randomly selected specimens were immersed in $33 \%$ sodium borate solution for $24 \mathrm{~h}$ and then carefully macerated. Axial projections of the dried skull and a special lateral projection of the two hemimandibles were taken using the previous regimen and film. The skull and the hemimandibles were placed directly on the radiographic films. 
All the radiograms were subjected to computerized cephalometric analysis. The radiographic image was digitized using of an optical reader (Fotovix II, Tamron Co.), and the measurements were obtained with DIRACOM3 software (LIDO, University of São Paulo). Angular measurements of deviation of the maxilla $(A M X)$ and mandible (AMD), with reference to a

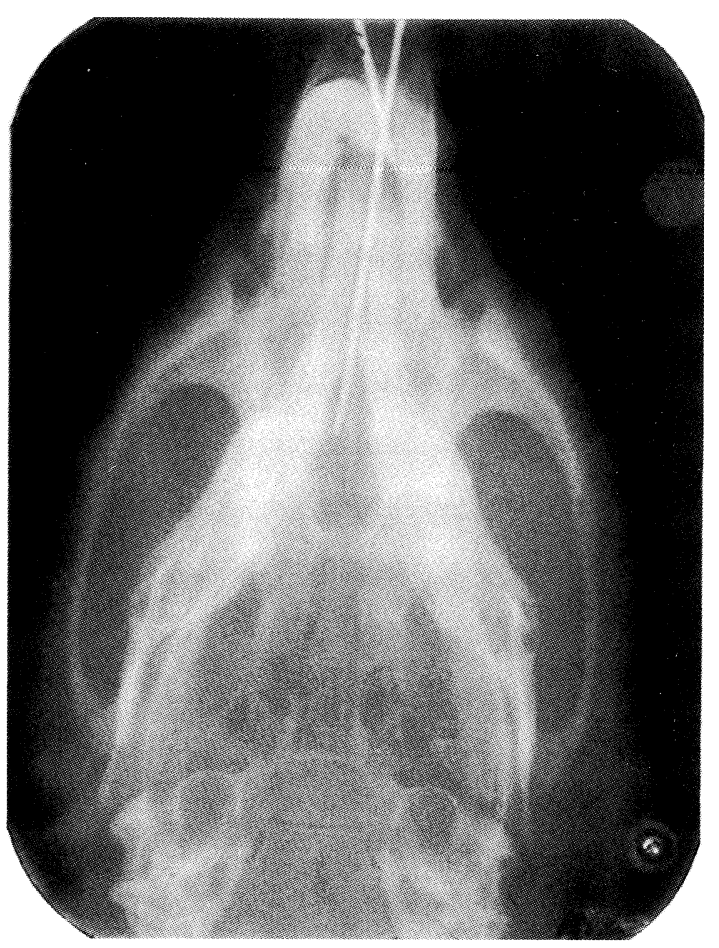

Fig. 1-A

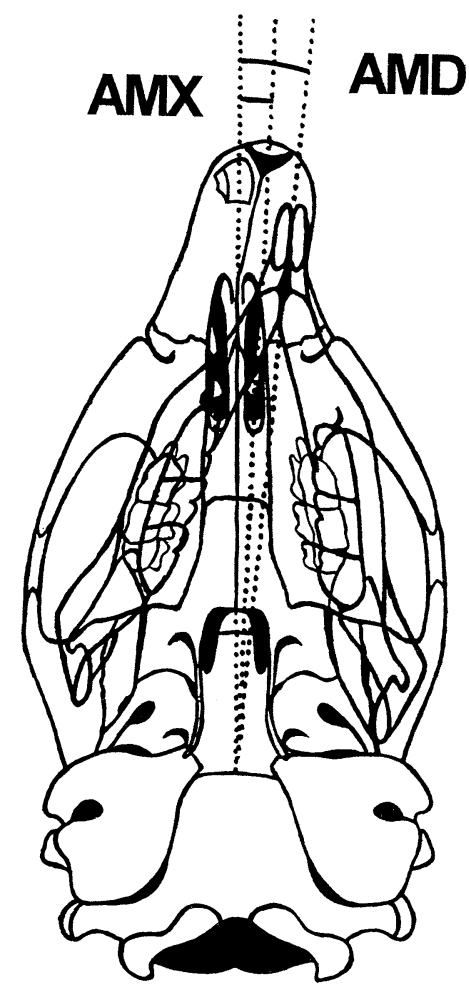

Fig. 1-B perpendicular line between both tympanic bullae, were registered on axial radiograms of fixed skulls (Fig. 1-A, B). The heights of both the ascending rami of the mandible were measured on rostro-caudal radiograms of fixed skulls, using the most external point of the zygomatic arch and anterior border of the antegonial notch as reference points (RRR and RRL) (Fig. 2-A, B).

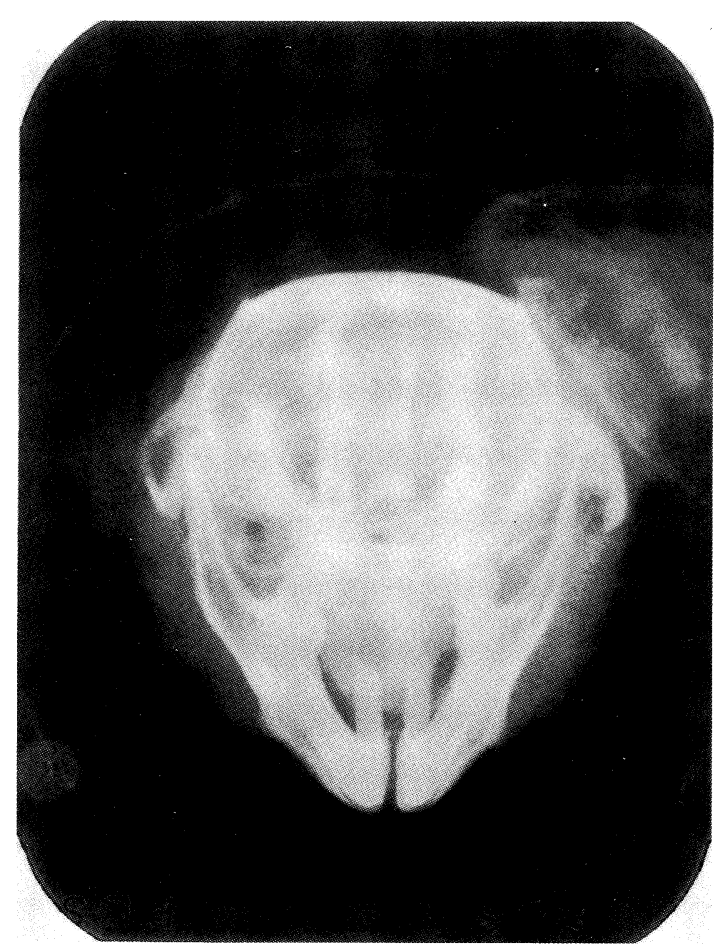

Fig. 2-A

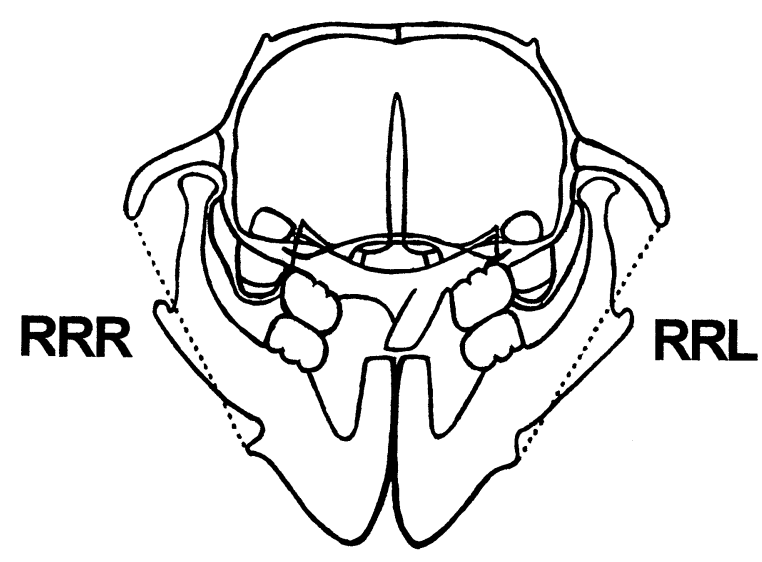

Fig. 2-B

Fig. 1-A Axial radiogram of a fixed skull. Short radiopaque line $=$ mesial surface of upper left incisor; long radiopaque line $=$ mandibular midline.

Fig. 1-B Angular measurements made.

Fig. 2-A Rostro-caudal radiogram of a fixed skull. Note deviation of the upper left incisor.

Fig. 2-B Linear measurements made. 
Using axial radiograms of dried skulls, the following distances were measured bilaterally: tympanic bulla and mesial root of the first molar (AS1); tympanic bulla and infraorbital foramen (AS2); and infraorbital foramen and incisal point (intersection of palatine midline and palatine surface of the upper left incisor) (AS3) (Fig. 3-A, B). On the lateral radiogram of the hemimandibles, the following lengths were measured: lower insertion of incisor to angular process (LM1); lower insertion of incisor to condylar process (LM2); angular process to condylar process (LM3); and intersection of the distal face of the third molar with the ascending ramus to antegonial notch (LM4) (Fig. 4-A, B). To evaluate the significance of differences between measurements on the left and right sides, Student's $t$ test was utilized. The level of significance was set at $\mathrm{p}<0.05$ for all statistical analyses.

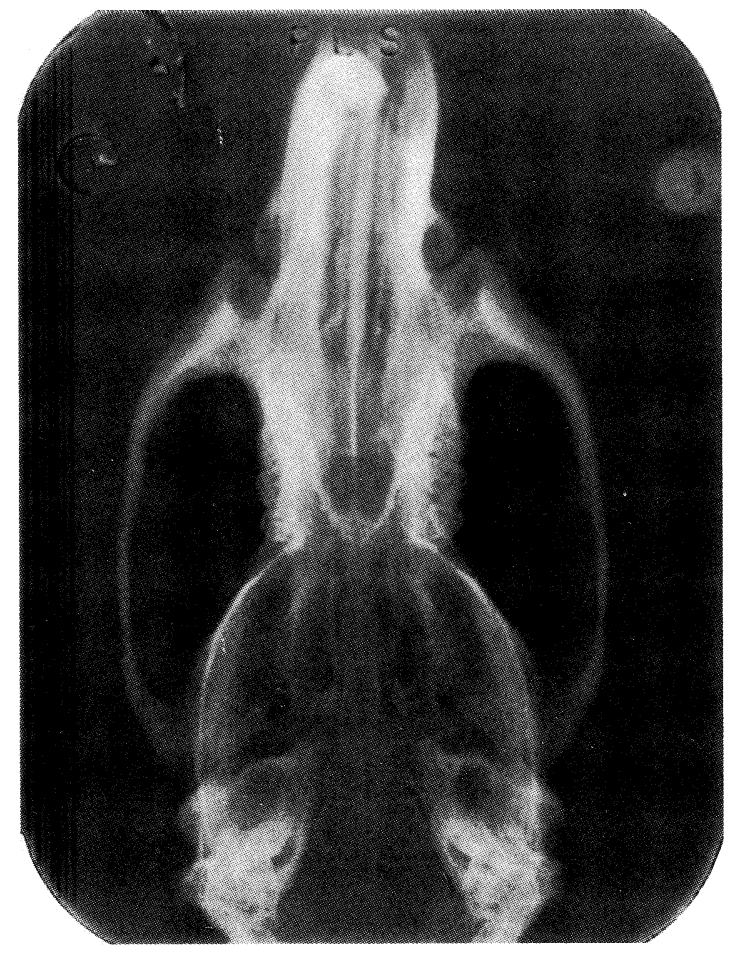

Fig. 3-A

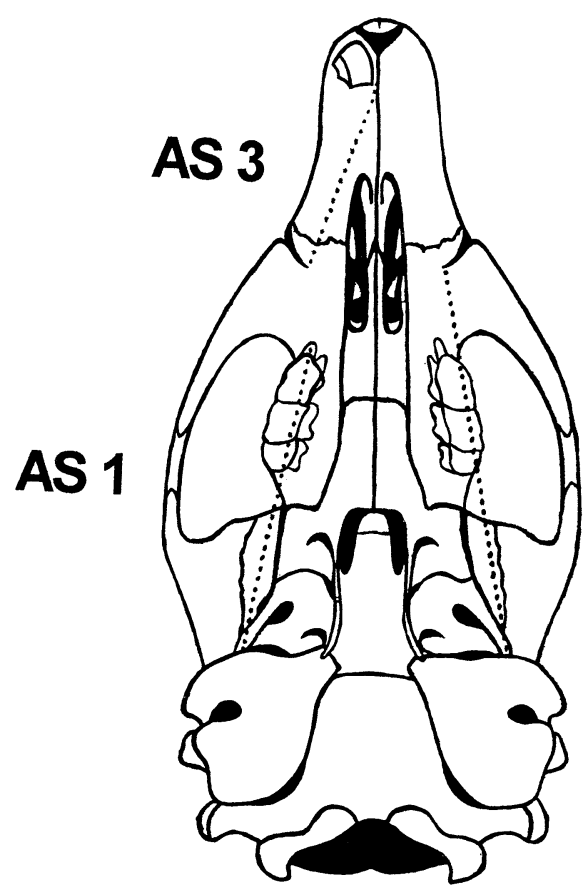

AS 2

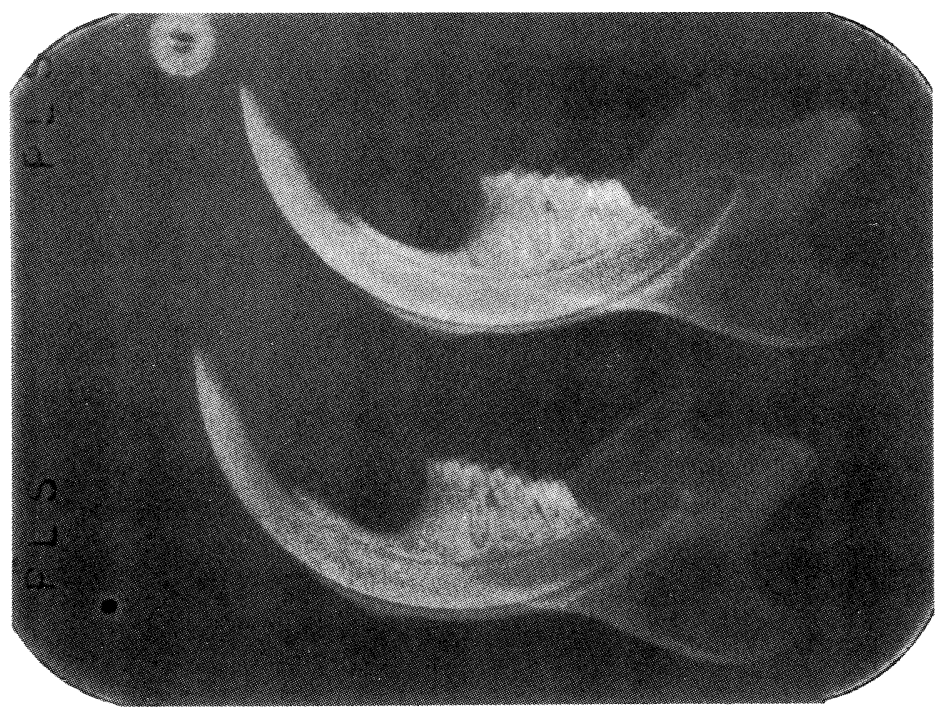

Fig. 4-A
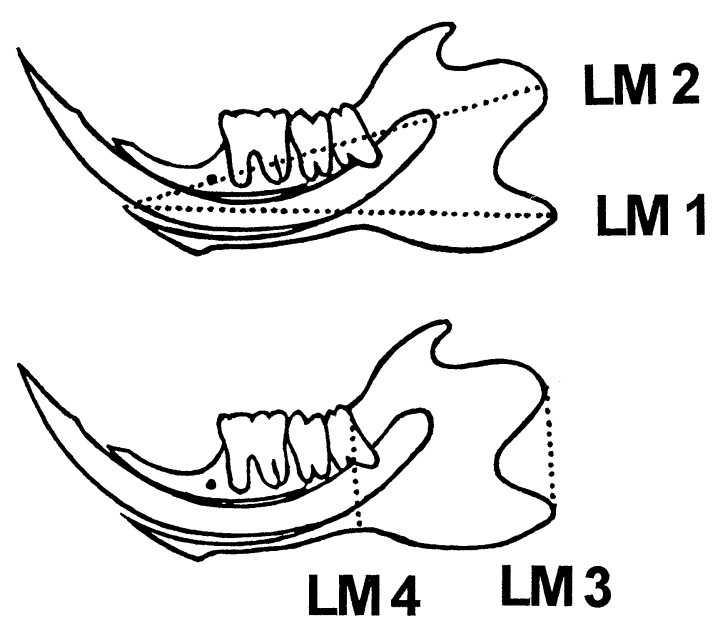

Fig. 4-B

Fig. 3-A Axial radiogram of a dried skull. Note deviation of the premaxilla to the right side.

Fig. 3-B Linear measurements made.

Fig. 4-A Lateral radiogram of hemimandibles.

Fig. 4-B Linear measurements made. 


\section{Results}

During the experimental period the animals ate without difficulty, and continued to grow and gain weight. Gross examination of the specimens at the end of the experiment revealed facial asymmetry, with deviation of the anterior portion of the maxilla to the right. The extraction site healed uneventfully, and the upper left incisor showed deviation toward the right. Signs of regular dental wear were seen on all the incisors. No noticeable changes in occlusion of the molar teeth were present. The mean values of angular measurements of deviation of the maxilla and mandible determined from the axial radiograms are shown in Table 1. We decided not to use these data due to their high standard deviation.

Table 1 Angular measurements from axial radiograms of fixed skulls

\begin{tabular}{ccc} 
& \multicolumn{2}{c}{ Measurements in degrees $(\mathrm{n}=35)$} \\
\hline Parameter & Mean & SD \\
\hline AMX & 3.94 & 4.12 \\
AMD & 0.65 & 4.04 \\
\hline
\end{tabular}

The mean heights of the ascending ramus of the mandible determined from rostro-caudal radiograms are shown in Table 2. There was no significant difference between the right and left sides.

Table 2 Height of the ascending ramus of the mandible determined from rostro-caudal radiograms

\begin{tabular}{ccc} 
& & Measurements in $\mathrm{mm}(\mathrm{n}=35)$ \\
\hline Parameter & Mean & SD \\
\hline RRR & 11.39 & 5.74 \\
RRL & 11.24 & 5.66 \\
\hline
\end{tabular}

The mean values of measurements determined from axial radiograms of dried skulls are shown in Table 3. There was a statistically significant difference between the right and left sides in AS3 ( $\mathrm{p}<0.05)$. The remaining parameters revealed no significant difference.

Table 3 Parameters of dried skulls

\begin{tabular}{ccccc} 
& \multicolumn{3}{c}{ Measurements in mm $(\mathrm{n}=10)$} \\
\hline \multirow{2}{*}{ Parameter } & \multicolumn{2}{c}{ Right side } & \multicolumn{2}{c}{ Left side } \\
\cline { 2 - 5 } & Mean & SD & Mean & SD \\
\hline AS1 & 16.74 & 8.38 & 17.17 & 8.60 \\
AS2 & 20.31 & 10.19 & 20.28 & 10.18 \\
AS3 & 7.61 & 3.85 & 8.50 & 4.26 \\
\hline * $<<0.05$ & & & &
\end{tabular}

The mean values of measurements from lateral radiograms of dried hemimandibles are shown in Table 4. There was no signficant difference between the right and left sides in any of the parameters.
Table 4 Parameters of hemimandibles

\begin{tabular}{ccccc} 
& \multicolumn{3}{c}{ Measurements in mm $(\mathrm{n}=10)$} \\
\hline \multirow{2}{*}{ Parameter } & \multicolumn{2}{c}{ Right side } & \multicolumn{2}{c}{ Left side } \\
\cline { 2 - 5 } & Mean & SD & Mean & SD \\
\hline LM1 & 23.73 & 11.90 & 23.93 & 12.01 \\
LM2 & 24.68 & 12.36 & 24.52 & 12.29 \\
LM3 & 8.09 & 4.06 & 7.98 & 3.99 \\
LM4 & 6.05 & 3.03 & 6.10 & 3.06 \\
\hline
\end{tabular}

\section{Discussion}

The present study has shown that unilateral extraction of the upper incisor from young rat results in facial asymmetry, due to significant reduction and deviation of the premaxilla on the same side. No significant alterations were observed in the rest of the maxilla. This finding indicates that unilateral extraction of an upper incisor produces impairment of facial growth localized in the incisor bone. Although there have been a number of studies on the effect of extraction of incisor teeth, the effect of unilateral upper incisor extraction has never been examined in experimental animals. Extraction of lower incisors reduces the symphyseal area and produces anterior rounding of the mandible $(8,9)$. When extraction is unilateral, a medial shift of the mandible has been described (1). These alterations, represented by shortening of the mandible, occur due to horizontal growth of the lower incisors (8). Extraction of the upper incisors produces shortening of the premaxilla $(8,9)$, due to vertical growth of the upper incisors (8).

We performed cephalometric measurements from radiograms and through computer analysis, and obtained versatile and reliable measurements, avoiding technical difficulties $(2,4)$. In this study the animals received an ordinary diet of rodent feed, avoiding any bias introduced by a soft diet. A more anteriorly directed growth direction, as well as changes in the shape of the mandible, have been related to the use of a soft diet in growing rats (4-6). The association of a soft diet with malocclusion has been reported to influence the development of masticatory organs (7). No significant alterations were observed in the mandible, confirming previous studies in which extraction of lower teeth caused impairment of mandibular growth (7-9). On the other hand, our results suggest that mensuration of the height of the ascending ramus from rostro-caudal radiograms would not be necessary when performing mensuration of hemimandibles (Tables 2 and 4). In our study, adaptation of masticatory function probably occurred. The remaining upper incisor presented rightward deviation, and there was regular dental wear on all the incisors. However, the findings related to the mandible may also be linked to its independent growth relative to the facial bones (10).

\section{References}

1. Castelli, W. A., Ramirez, P. C. and Burdi, A. R. (1971) Effect of experimental surgery on mandibular growth in Syrian hamsters. J. Dent Res. 50, 356-363 
2. Del Campo, A. F., Elizondo, M. M.; Magnelli, L. M., Valadez, A. S. and Ontiveros, D. S. (1995) Craniofacial development in rats with early resection of the zygomatic arch. Plast. Reconstr. Surg. 95, 486495.

3. Duker, J. and Harle, F. (1974) The influence of unilateral operations, with reference to the soft tissue only, on the growth of the skull. J. Max.-Fac. Surg 2, 44-48

4. Kialiaridis, S., Engstrom, C. and Thilander, B. (1985) The relationship between masticatory function and craniofacial morphology. I. A cephalometric longitudinal analysis in the growing rat fed a soft diet. Eur. J. Orthod. 7, 273-283

5. Kiliaridis, S. (1986) The relationship between masticatory function and craniofacial morphology. III.
The eruption pattern of the incisors in the growing rat fed a soft diet. Eur. J. Orthod. 8, 71-79

6. Yamada, K. and Kimmel, D. B. (1991) The effect of dietary consistency on bone mass and turnover in the growing rat mandible. Arch. Oral Biol. 36, 129-138

7. Kuboyama, N. and Moriya, Y. (1995) Influence of diet composition and malocclusion on masticatory organs in rats. J. Nihon Univ. Sch Dent. 37, 91-96

8. Riesenfeld, A. (1969) The adaptative mandible: an experimental study. Acta Anat. 72, 246-262

9. Ranta, R., Alhopuro, S. and Ritsila, V. (1973) The effect of tooth extractions on the growth of the jaws in rabbits. Proc. Finn. Dent. Soc. 69, 118-119

10. Enlow, D. H. (1990) Facial growth. 3rd ed., W. B Saunders, Philadelphia 